\title{
Energy transfer in supramolecular materials for new applications in photonics and electronics
}

\author{
Ken-Tsung Wong ${ }^{1}$ and Dario M Bassani ${ }^{2}$
}

Supramolecular materials use self-assembly of molecular components to form complex architectures that may otherwise be extremely difficult to prepare. One of the fundamental aspects of this approach is that relatively weak intermolecular forces are used to direct the assembly of the subcomponents. An important point is how to achieve strong electronic communication throughout the material in view of the 'looseness' of the molecular constituents, which interact only weakly. This is particularly important for applications in molecular electronics where exciton delocalization and charge transport generally limit the overall device performance. This review focuses on recent advances in supramolecular materials and architectures that are engineered to possess efficient energy transfer between the self-assembled component in view of new applications in photonics and electronics.

NPG Asia Materials (2014) 6, e116; doi:10.1038/am.2014.53; published online 25 July 2014

\section{INTRODUCTION}

Supramolecular self-assembly is the process by which two or more molecular components spontaneously organize into an ordered architecture, and is routinely used by nature to build up the molecular machinery used to perform the complex tasks necessary in living organisms. From a practical perspective, it provides an elegant solution to explore the limits situated between the top-down technological miniaturization approach and the bottom-up construction of single-molecule devices. Its principal advantage arises from the use of smaller subunits that can be more easily prepared, characterized and tuned. This also opens up prospects for materials that selfrepair or that autonomously regulate their function in response to the operational conditions. ${ }^{1-6}$

Aside from the complexity of designing molecules that spontaneously self-assemble into ordered structures, the issue of obtaining a functional response from the supramolecular architecture that presents an added value with respect to the properties of the individual molecular constituents must be considered. In its absence, there may be no need or benefit in using self-assembly in the fabrication of a molecular device compared with the use of a mixture of the individual components. To elicit a collective response in a molecular assembly, the notion of molecular communication has been introduced in order to quantify the extent of the electronic and/or steric interactions between molecular subunits. As molecules possess a localized electron density distribution, they exhibit relatively sharp electronic transitions and reduced intermolecular electronic overlap integrals except in specific cases, for example, when $\pi$-stacking is important. As a result of this, molecule-based electronic materials are inherently much less susceptible to defects and impurities as their effect is localized to nearby molecules only. However, the lack of intermolecular electronic communication also reduces the charge carrier mobility and exciton delocalization. Supramolecular interactions, as shown in the following pages, can be used to direct how molecules self-assemble and thus enhance (or reduce, as needed) electronic interactions.

The underlying principle behind the use of designed intermolecular interactions in organic devices is to dissociate the fundamental electronic properties of the molecular component from its selfaggregation properties that drive nonspecific association and would otherwise control the morphology of the aggregates and the active layer. The latter are important in determining energy and electron transfer as a consequence of through space interactions between molecules in the solid phase. Thus, independent control of the morphology and the electronic properties of the molecular subunits will make their optimization possible by considering each process separately. As a result of the dynamic nature of the self-assembly process, the deposition and post-deposition treatment (annealing) can have profound influence on the overall properties. ${ }^{7}$ Depending on the degree of order within the material, different theoretical models can be used to describe energy transfer processes and the mobility of charge carriers, from band theory for structurally ordered materials, to tight-binding models for weakly disordered systems, and hopping models for localized charges in strongly disordered materials. Grozema and Siebbeles ${ }^{8}$ provided an overview of charge-transport models applicable to self-organizing molecular materials.

Supramolecular interactions can benefit from any combination of intermolecular forces, ranging from weak hydrophobic/dispersion forces, to hydrogen-bonding ( $\mathrm{H}-\mathrm{B})$ or relatively strong metal ion coordination. The energies of these interactions span a wide range, from $<1$ to $4 \mathrm{~kJ} \mathrm{~mol}^{-1}$ for relatively weak hydrophobic or aromatic 
$\pi$-stacking interactions and single-point $\mathrm{H}$-B, to $>250 \mathrm{~kJ} \mathrm{~mol}^{-1}$ for very strong coordinative bonds. Besides the free energy, other factors such as kinetic lability and directionality are also important in characterizing supramolecular forces. For example, some (for example, H-B and coordination bonds) are directional, others only partly directional (for example, $\pi$-stacking), while others are non-directional (dispersive forces). Clearly, directionality is desirable in cases where a bottom-up design is expected to lead to the formation of precise, well-defined architectures. For these applications, the use of $\mathrm{H}-\mathrm{B}$ and coordinative bonds has been privileged, with $\mathrm{H}-\mathrm{B}$ interactions being particularly well-suited toward the construction of photoactive supramolecular assemblies as they do not absorb in the ultraviolet and visible range. Aromatic $\pi$-stacking, a principal driving force behind the formation of discotic liquid crystals, ensures moderate to good electronic communication between the molecular components. ${ }^{9,10}$ To build complex assemblies comprising several distinct subunits, it can be necessary to combine different supramolecular interactions that can operate independently. These should be orthogonal in their binding to allow sequential assembly with error correction as the system explores all possible low energy architectures. ${ }^{11}$ When the electronic interactions are sufficiently strong or driven by external forces, ${ }^{12}$ the formation of $J$ - or $H$-type aggregate structure and their subsequent effect on the electronic absorption and emission spectra is observed. The scope of this article is to discuss the more recent advances in the use of supramolecular architectures to controlling energy transfer in organic self-assembled materials, with particular emphasis on the development of new applications such as sensing and electroluminescent materials. Other potential uses, such as electron transfer for solar energy conversion, have been the subject of numerous recent reviews. ${ }^{13-16}$

\section{ENERGY TRANSFER IN ORDERED SUPRAMOLECULAR AGGREGATES}

Energy transfer, also known as exciton hopping in the solid, involves the transfer of electronic excitation energy from one chromophore to another. Two principal mechanisms, one based on resonance between the electronic transition dipole moments of the donor and the acceptor (Förster energy transfer) and the other on an electron exchange mechanism (Dexter energy transfer) are possible. Both require spectral overlap between the emission of the donor and the absorption spectrum of the acceptor and, whereas the latter relies on close contact between the donor and the acceptor, the former is still efficient even at longer distances. In extended supramolecular aggregates, such as micelles, vesicles or gels, the molecular components are organized in a solid or semisolid framework, akin to a solid environment. In this case, long-range energy transfer (several nanometers) can take place by hopping between chromophores. This phenomenon is also operational in organic photovoltaic materials, where hopping distances of $c a 10 \mathrm{~nm}$ have been determined, ${ }^{17}$ and similar hopping distances were found in helical $\pi$-stacks of perylene tetracarboxydiimide (PDIs). ${ }^{18}$ The dynamic nature of supramolecular assemblies further allows their interconversion between distinct morphologies by external stimuli ${ }^{19}$ and allows the formation of hybrid inorganic-organic frameworks. ${ }^{20}$

Molecular gels are formed by strongly anisotropic growth along a preferential direction during self-assembly, leading to an intertwined fiber network, which traps solvent molecules to form a gel. As unidirectional growth can often be achieved by the incorporation of a linear geometry of complementary supramolecular interactions (for example, H-B), gel materials have been a common scaffold used to induce ordering of $\pi$-functional materials. ${ }^{21,22}$ This area has been the topic of recent thorough reviews by Babu et al. ${ }^{16,23}$ and will not be discussed in detail except for the generation of white light from aggregates using energy transfer where it can be sometimes possible to follow the energy transfer across multiple chromphores in the assembly. This is the case in an acene-based organogel composed of a blue light-emitting anthracene donor co-assembled with tetracene and rubrene acceptors emitting in the green and red region, respectively. ${ }^{24,25}$ Upon excitation of the anthracene donor, energy transfer populates the excited states of green and red emitters that are dispersed into the matrix (1.2\% red and green acceptors), which contribute to the emission. Despite their low concentration, energy transfer from the green to the red component was also evidenced, supporting a previous observation of long ( $>100$ chromophores) energy hopping in such materials. ${ }^{26}$ Similar results were achieved in a bi-component system using charge-transfer interactions between naphthalene and 1,2,4,5-tetracyanobenzene to assemble rigid tubes. Addition of a small amount of pyrene affords white light emission through energy transfer (Figure 1). ${ }^{27} \mathrm{~A}$ selection of representative systems operating along such lines have been recently reviewed by Armaroli and co-workers, ${ }^{28}$ and the occurrence of long-range excitation energy migration in self-assembled fibers opens up new opportunities in the construction of amplified molecular sensors. ${ }^{29}$ Their operation would be similar to the quenching mechanism in fluorescent conjugated polymers, whereby the exciton migrates along a polymer chain to explore multiple binding sites. ${ }^{30}$ This approach was used by Ajayaghosh and co-workers ${ }^{31}$ to propose a fluorescent molecular gel capable of detecting electron-deficient aromatics (such as TNT) with attogram sensitivity. The scaffold of the material is composed of a $\pi$-conjugated tri-alkoxyphenylene-vinylene, appended with pentafluorophenyl groups that impart phase separation through hydrophobic forces and $\mathrm{C}-\mathrm{F} \cdots \mathrm{H}-\mathrm{C}$ interactions. X-ray diffraction patterns confirm a regular packing of the chromophores that is maintained upon exposure to nitroaromatic compounds. This point is essential as the packing is crucial for long-range exciton diffusion, which allows the amplification of the signaling ability of the material.

Energy transfer between two compartments in an aggregate was demonstrated by Würthner and co-workers ${ }^{32}$ by placing a $\mathrm{pH}$ sensitive fluorophore in the interior of a vesicle comprising a luminescent shell. The latter was polymerized to maintain the structural rigidity of the assembly, thus locking the PDI red light emitters in place. A bis-pyrene chromophore whose emission can be switched from monomer (blue emission) to excimer (green emission) by the $\mathrm{pH}$ was sequestered inside the vesicle with the aim of absorbing incident radiation and emitting blue-green fluorescence. Förster radiative energy transfer from the fluorophores inside the vesicles to the chromophores located on the peripheral shell thus results in aggregates whose emission envelope is $\mathrm{pH}$-tunable and, at $\mathrm{pH}=9$, the emission from the single aggregates appears white to the eye. This system therefore displays emission that can be localized with submicron precision and that is responsive to outside stimuli. There is great appeal for the use of vesicles (or micelles) ${ }^{33}$ as adjustable point light sources, but their preparation can be cumbersome and not easily compatible with the fabrication of luminescent devices. Generally, long alkyl chains and a watersoluble segment are required for inducing amphiphilicity and controlling the surface curvature of the aggregates, which are formed by dispersion of an organic solution into an aqueous phase. ${ }^{34}$

To circumvent problems associated with the amphiphilic nature of the supramolecular assembly components in micelles and vesicles, it is possible to use polymeric assemblies (polymersomes) or compounds specifically designed to adopt vesicle-like architectures based on 
a

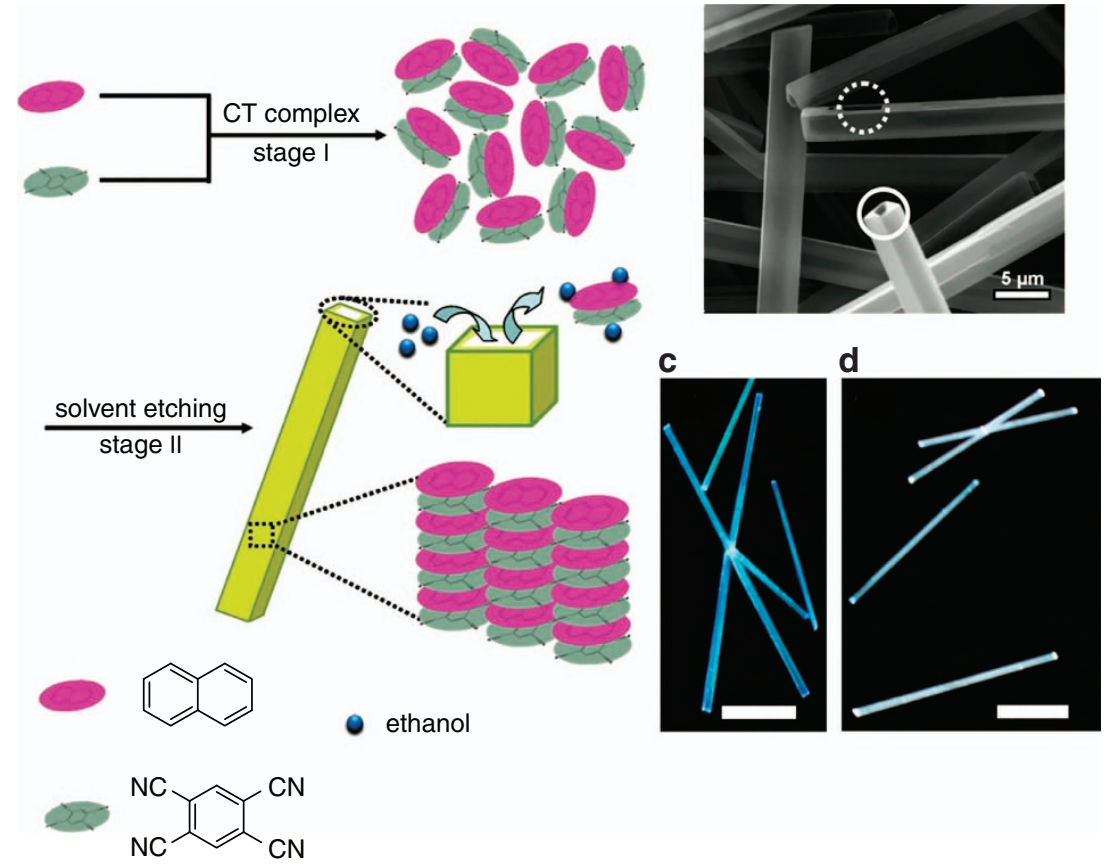

Figure 1 In acetonitrile solution, naphthalene and 1,2,4,5-tetracyanobenzene form charge-transfer (CT) complexes that self-assemble into hollow rods when the solution is dispersed in an ethanol-water mixture (a). Scanning electron microscope image of the rods (b) evidences their hollow nature. Upon excitation $(330-380 \mathrm{~nm})$, blue emission from the CT complex is observed (c). Addition of pyrene $(0.015 \%)$ results in the emission of white light through an energy transfer process in the self-assembled rods (d, scale bars are $30 \mu \mathrm{m}$ ). Adapted with permission from reference Lei et al. ${ }^{27}$

designed supramolecular interactions. For example, it is well known that urea and bis-urea, as well as analogous ureopyrimidones selfassemble into fibers because of the unidirectional nature of their binding site. Instead, biuret adopts a cyclic structure to expose two $\mathrm{H}-\mathrm{B}$ sites that are orthogonal to each other, and hence assembles into $\mathrm{H}-\mathrm{B}$ sheets (Figure 2a). In solution, such sheets are subjected to continuous fluctuations and possess vacant peripheral binding sites. They eventually fold into spherical assemblies to reduce exposed vacant $\mathrm{H}-\mathrm{B}$ sites (Figure $2 \mathrm{~b}$ ). In fact, the directionality of $\mathrm{H}-\mathrm{B}$ interactions allows a relatively straightforward interpretation and projection of possible architecture topologies, including switching between the formation of fibers and spheres $^{35}$ or crystals and vesicles $^{36}$ or spheres. ${ }^{37}$

Many rigid luminescent chromophores can be substituted for the photoactive core in Figure $2 \mathrm{~b}$ without altering the formation of the vesicle-like assemblies. This allows tailoring of the photophysical as well as the photochemical properties of the material independently and of the final morphology of the aggregates. In fact, such hollow spheres were shown to form spontaneously upon dissolution of the solid into an aprotic solvent that does not impede H-B. In such cases, the presence (or absence) of water does not influence the formation of the artificial vesicles as their molecular components are pre-programmed to self-assemble according to specific interactions and do so in solution before casting onto various substrates. Indeed, the presence of hollow spherical aggregates possessing similar size as the aggregates observed by microscopy could be evidenced by small-angle neutron scattering. ${ }^{40}$

By tailoring the absorption and emission envelope of the luminophore, it is possible to obtain luminescent spheres that emit light of a given wavelength. In the case of oligo-fluorenes (BU-1), the resulting emission is not affected by aggregation through $\pi$-stacking, which would otherwise induce a strong red shift in the emission spectrum because of the formation of J-aggregates. Rather, the emission is strongly dependent on the presence of small amounts of dopants that can be excited through energy transfer processes. By using dopants consisting of a chromophore whose absorption envelope overlaps with the emission envelope of the donor, and that also respects the supramolecular algorithm for incorporating into vesicles (that is, a rigid core appended with biuret units on either end), the materials can be dispersed in the aggregates without disrupting the supramolecular topology. In this case, the dispersion of colors obtained was found to be very uniform and not to vary between aggregates as would be expected for an anisotropic distribution of acceptors. Even for small quantities of dopants (for example, $0.20 \mathrm{~mol} \%$ of BU-2 and $0.25 \mathrm{~mol} \%$ of $\mathbf{B U}-3$ ), the emission from the donor is substantially decreased (only accounting for $85 \%$ of the global emission despite accounting for $\mathrm{ca} 99.9 \%$ of the absorbed light) and emission from the acceptor components is strong (5\% and $10 \%$ for BU-2 and BU-3, respectively).

Stable lamellar assemblies of organic chromophores can be readily obtained by grafting them onto flat nanoscopic inorganic structures. Rao et al. ${ }^{41}$ thus showed that it is possible to use a cationic clay to assemble anionic fluorophores in an ordered architecture. The chromophores were carboxylic or sulfonic acid derivatives of coronene or sulforhodamine $G$, which were assembled onto an amino-functionalized clay based on an layered magnesium phyllo(organo)silicate having a 2:1 trioctahedral smectite-like structure. The coronene was used as the blue-emitting donor component, whereas the rhodamine $\mathrm{G}$ acted as the energy-accepting yellow emitter. Soft gel-like aggregates are formed at total concentrations of donor and clay components greater than ca 8 wt.\%. Upon inclusion of a small amount of acceptor component (0- 
a

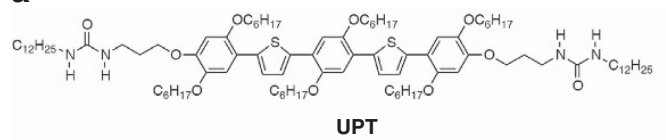

UPT
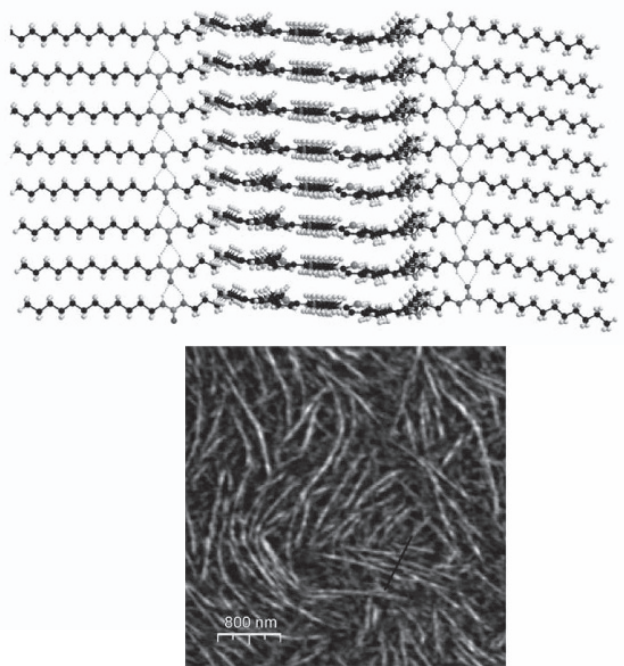

b

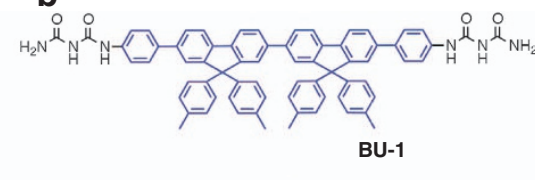

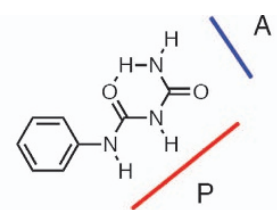
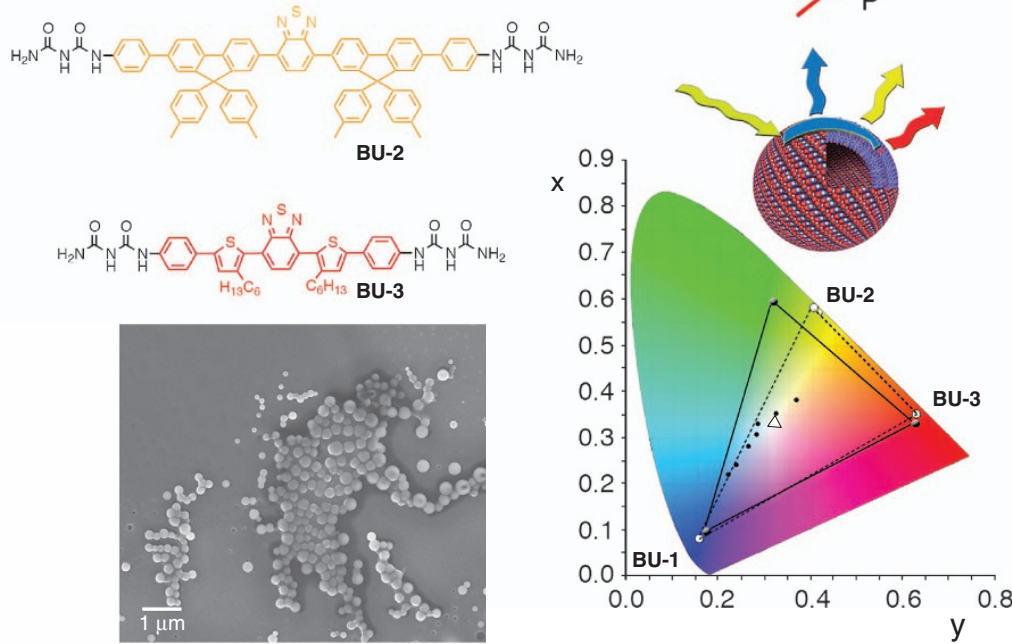

Figure 2 (a) It is often the case that two urea residues appended to a $\pi$-conjugated core, such as a phenylenethiophene unit (UPT), induce the formation of stacked assemblies and the formation of fibrilar gels (crystal structure and atomic force microscopy image below). Adapted with permission from reference Dautel et al. ${ }^{38}$ (b) The use of two biuret hydrogen-bonding (H-B) units code for the spontaneous formation of hollow spheres in solution that persist upon solvent evaporation (scanning electron micrograph, bottom). This is attributed to the existence of two $\mathrm{H}$-B sites on each biuret unit that are orthogonal to one another (labeled A and P). Compounds BU-1, BU-2 and BU-3 emit in the blue, yellowish green and red region of the spectrum and can be combined in a single vesicle to produce light of any color contained in the dashed triangle. Adapted with permission from reference Tseng et al. ${ }^{39}$

$1 \mathrm{~mol} \%$ ), the emitted light gradually changes from blue to yellow, and white light is obtained for a concentration of acceptor $=0.65 \mathrm{~mol} \%$. The gels thus obtained are stable and free-standing, making the system interesting for lighting applications. H-B between complementary recognition sites can also be used to prepare extended assemblies by using ditopic subunits to allow the formation of extended architectures, such as for cyanuratemelamine ribbons appended with PDI chromophores. ${ }^{42}$

It is quite reasonable to assume that intra-aggregate energy transfer occurs non-isotropically within the sample, and that this can also depend on the presence of islands of acceptors because of microscopic phase separation within an individual aggregate. To investigate this point, Schenning, Herz and co-workers ${ }^{43}$ investigated energy transfer efficiency as a function of donor/acceptor ratios and found that this is non-linear for acceptor ratios $>c a 0.5 \%$ because of clustering of the acceptors within the nanoparticles. It should be noted that this value is likely to depend on the molecular constituents, but that the approach used to highlight acceptor separation within the aggregate is quite general. ${ }^{44}$ The availability of a confocal microscope with which to examine the emission from individual aggregates further enables the investigation of variability between different single aggregates that may arise from non-equilibrium dynamics during aggregate formation. An analogous situation was encountered in surfacegrafted polymer particles appended with fluorescein fluorophores, where excitation migrates between identical fluorophores until it trapped by a non-fluorescent aggregate. ${ }^{45}$ In the case of PDIs, a wellknown class of chromophores that are prone to forming $\pi$-stacked aggregates, relaxation of the molecules surrounding the localized excited state can provide stabilization that leads to the formation of a potential energy well at that location. ${ }^{46}$ Localization of the excitation energy onto a single chromophore effectively traps the exciton and reduces energy transfer.

Understanding intra-aggregate energy migration and Förster Resonance Energy Transfer processes is also of interest in cases where such processes are undesirable. This would be the case in spatially colocalized distribution of emitters of different colors, such as in the voxels (volumetric pixels) used in color display devices. In these systems, the individual primary colors are turned on to produce a composite color as perceived by the human eye and it is important that cross-contamination of the primary colors is avoided. This problem is particularly acute in organic light-emitting diode displays, where the chromophores are evaporated through a mask. By taking advantage of the large Stokes shift induced by an excited-state proton transfer process in a series of ortho-hydroxyphenylimidazoles, Park and co-workers ${ }^{47}$ showed that it is possible to impede energy transfer by reducing the spectral overlap between the molecular component in a multichromphoric assembly and were able to produce a mixture of individual molecular producing RGB emission without cross-talk (Figure 3).

\section{ENERGY TRANSFER IN MULTICOMPONENT MOLECULAR CRYSTALS}

Energy transfer in organic crystals is a wide topic spanning chemistry and physics that is much too broad to be discussed in detail here. Instead, attention will be focused on examples of systems able to use supramolecular interactions to combine several chromophores in a regular 'crystalline' arrangement in the solid. For comparison, in an archetypical organic crystal such as rubrene, exciton delocalization was estimated by Moran and co-workers ${ }^{48}$ to be $c a 18$ molecules through a combination of spectroscopic techniques and modeling of 
a

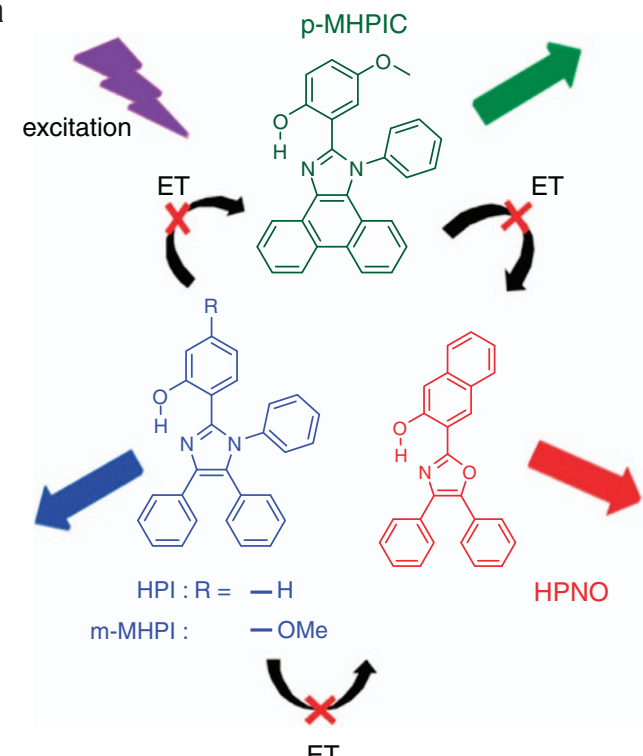

b

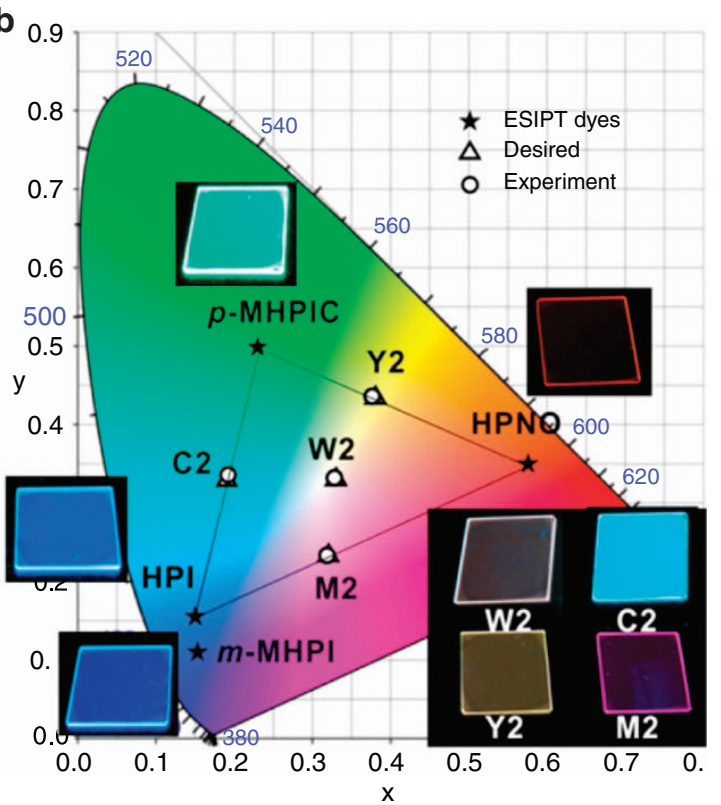

Figure 3 Excited-state intramolecular proton transfer (ESIPT) in a series of o-hydroxyphenylimidazoles (a) induces a large Stokes shift in these materials, which effectively reduces the overlap integral between the emission of the donor and the absorption of the acceptor components. This turns off energy transfer, making these materials interesting for obtaining colors that are the binary mixture of the chemical components (b). Adapted with permission from reference Kwon et al. ${ }^{47}$ Copyright 2013 the American Chemical Society.

the spectral densities. These techniques allow the determination of the electronic relaxation dynamics, which in turn govern coherent energy transfer within the crystal. Hayward and co-workers ${ }^{49}$ reported the self-assembly of crystals of poly(3-hexylthiophene) onto perylene tetracarboxydiimide (PDI) crystals. The former grows as small hairs onto the larger fiber-like crystals and acts as quenchers of the intense PDI emission. It is proposed that the thiophene polymer behaves as an additive to enhance the PDI solubility and reduce the lateral dimensions of the PDI crystals, which can be tuned from 25 to
$200 \mathrm{~nm}$. A qualitative comparison of the emission from the PDI nanocrystals with and without thiophene polymer crystals shows that the fluorescence is nearly completely quenched, as would be expected for efficient energy transfer within the PDI crystals.

The use of designed supramolecular interactions (as opposed to, for example, intrinsic crystal packing forces) can be applied to favoring the formation of otherwise hard-to-reach polymorphs. When growth along with one of the crystal axes is privileged, then fibrilar or needle-like crystals are obtained. Spontaneous branching then leads to the formation of arborescent architectures, such as those reported by Nabiev and co-workers ${ }^{50}$ for core-shell nanoparticles. Time-resolved photoluminescence studies indicated that some of these structures show energy transfer from the periphery to the interior of the dendritic core. Chain branching was also evident in soft $\mathrm{C}_{60}$ fullerenebased materials reported by the Nakanishi group. ${ }^{51}$ In this case, the formation of various fullerene morphologies, including lamellar structures conducive toward two-dimensional energy and electron transport, were obtained by the use of branched alkyl chains (Figure 4).

As an alternative to hydrophobic forces, $\mathrm{H}-\mathrm{B}$ can be used to induce formation of well-defined fullerene assemblies. These may (or not) also possess saturated hydrocarbon chains to assist the self-assembly and improve solubility, although a high proportion of saturated hydrocarbon chains can be deleterious to energy and electron transport. Barbituric acid derivatives possess two H-B sites, which are partially self-complementary through the formation of two N-H... O H-bonds. Thus, a fullerene-barbituric acid adduct was found by $\mathrm{Chu}$ et al. $^{52}$ to self-assemble into $\mathrm{H}$-bonded ribbons in which the fullerenes are in close contact along the H-bonded tape-like architecture (Figure $4 \mathrm{~b}$ ).

In the supramolecular fullerene material depicted in Figure $4 \mathrm{~b}$, there are two equivalent fullerene molecules per unit cell, and it would be reasonable to assume that energy transfer processes are limited to exciton diffusion amongst identical sites. However, the particular photochemical behavior of fullerenes, along with the specific molecular arrangement directed by the H-B interactions of the barbiturate motif, can strongly influence the photophysical properties of the material. In crystalline $\mathrm{C}_{60}$ samples, excitons formed upon absorption of light by a single fullerene are delocalized over two (or more) fullerenes. ${ }^{52}$ The resulting excimer (for excited state dimer) is slightly stabilized with respect to the locally excited state, and therefore emits at a longer wavelength than is observed for an isolated $\mathrm{C}_{60}$ molecule. However, when a fullerene pair is excited, photoinduced dimerization across the proximal 6,6 double bonds is possible. This can take place in the solid, but also in solution when two fullerenes are held in proximity due to supramolecular interactions as barbiturate $\mathrm{H}-\mathrm{B}$ motifs are well-suited to promoting $[2+2]$ photocycloadditions. ${ }^{54}$

Fluorescence from isolated fullerenes is not polarized because of the high symmetry of the sphere $\left(\mathrm{I}_{h}\right)$, unlike what is usually observed for aromatic hydrocarbons that emit light, which is polarized along the long and short axes of the chrophophore. In the solid, emission from fullerene excimers can be polarized because of their reduced symmetry with respect to fullerene. In the case of emission from the fullerene ribbons in Figure 4c, the emission was found to be polarized with a maximum intensity at $60^{\circ}$ with respect to the long axis of the H-bonded ribbon. ${ }^{55}$ This was interpreted as arising from fast exciton migration to populate the lowest energy of the three possible distinct fullerene excimer pairs that can form in the solid state. Interestingly, this excimer is also photoreactive and undergoes photoinduced dimerization upon prolonged irradiation. As a consequence, the electronic energy of this state is raised above that of the other 


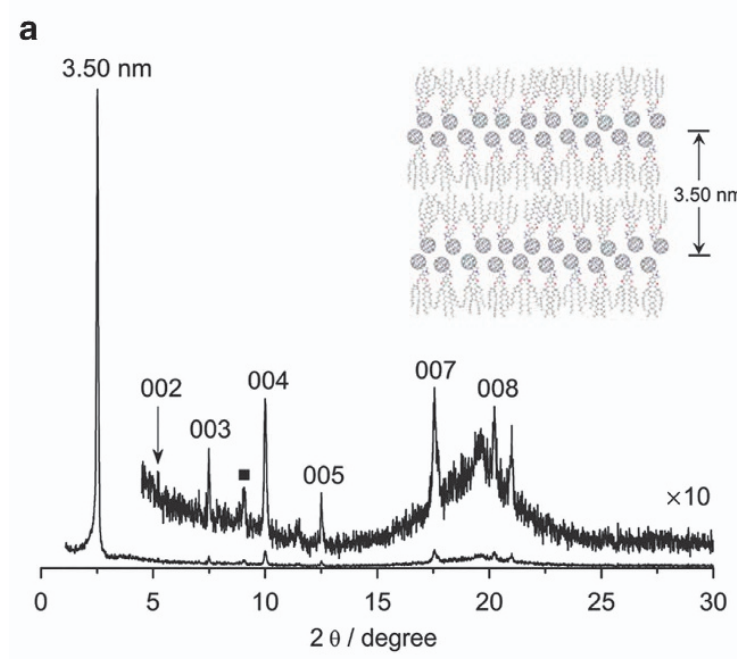

C

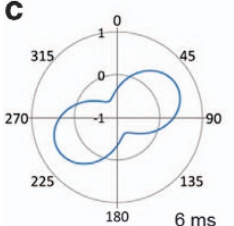

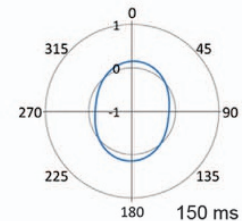

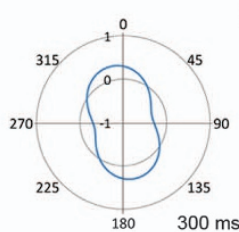

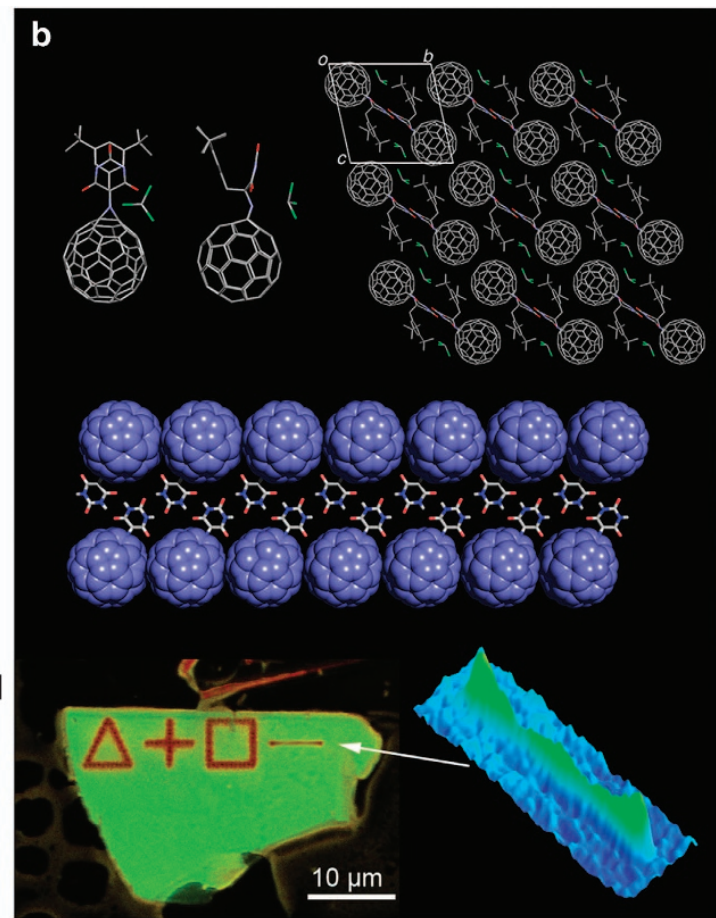

Figure 4 (a) The presence of branched alkyl substituents can generate lamellar structures in which the fullerene moieties are in closed contact as evidenced by the X-ray diffraction (XRD) spectrum. Reproduced from reference Li et al..$^{51}$ with permission of The Royal Society of Chemistry. (b) A similarly linear arrangement of fullerenes can also be obtained by designed hydrogen-bonding $(\mathrm{H}-\mathrm{B})$ interactions as observed in the crystal structure of a fullerenebarbiturate derivative in which energy transfer is particularly efficient. Reproduced with permission from reference Chu et al.52 Copyright 2010 the American Chemical Society. Upon irradiation, the polarized emission from this material undergoes a switch in direction (c) of $\mathrm{ca} 60^{\circ}$ without any changes in the emission spectrum, intensity or lifetime. This process, termed photopolism, can be used to write information that can only be read out through the polarization component of the emission (d). Reproduced with permission from reference Raffy et al. ${ }^{53}$

excimer pairs, and energy transfer now populates a different excimer whose electronic transition dipole is oriented differently from that of the first. The result is that of a material that emits polarized light, which, upon excitation, undergoes a rotation of the polarization without any changes in its emission spectrum, intensity or lifetime. This process was termed photopolism by the authors, and relies on the combination of efficient energy transfer and photoinduced reactivity in the solid. ${ }^{53}$ The process is diffraction limited and can be used to write information on the material that can only be detected by the use of fluorescence polarization.

\section{ENERGY TRANSFER IN WELL-DEFINED SUPRAMOLECULAR ASSEMBLIES}

Supramolecular fullerene assemblies have attracted considerable interest in view of their use in solar energy conversion. ${ }^{56,57}$ In this case, it is charge separation (that is, electron transfer) that is pursued, but energy transfer processes are often also present as either preceding charge separation or as a competitive process. ${ }^{58}$ For example, intramolecular energy transfer in a zinc-phthalocyanine/porphyrin dyad was found to be extremely fast, occurring on a sub-picosecond time scale. ${ }^{59}$ This allowed light harvesting by the phthalocyanine, which powered the subsequent electron transfer from the excited porphyrin to the phthalocyanine. The overall result is an accrued efficiency of photocurrent when the dyad was self-assembled on a transparent $\mathrm{TiO}_{2}$ electrode by slowing charge recombination on the electrode surface as previously suggested by Imahori and coworkers. $^{60}$ Sequential energy-electron transfer was also observed when the phthalocyanine-porphyrin dyad was non-covalently attached to a fullerene by axial metal ion coordination, ${ }^{61}$ and in an analogous system using an additional bodipy fluorophore. ${ }^{62}$ Control over energy vs electron transfer processes in the well-studied porphyrin-fullerene architectures can generally be obtained by varying the solvent polarity, with non-polar environments favoring energy transfer whereas more polar solvents will accelerate electron transfer by stabilizing the ensuing radical ion pair that is generated. Threepoint $\mathrm{H}-\mathrm{B}$ was used by Rotello and co-workers ${ }^{63}$ to assemble fullerenes on the periphery of suitable modified quantum dots. The photophysical properties of the material evidence ultra-fast charge separation, but it is reasonable to assume that it is also possible to steer the system toward energy transfer by adjusting the relative energy levels of the donor and acceptor.

PDIs are known for their propensity to aggregate, as mentioned in the section on supramolecular aggregates, but they can also be used in structurally well-defined assemblies. Thus, in a large molecular square comprising 16 pyrene donors and 4 PDI acceptors (Figure 5a), a fast energy transfer rate of $5 \times 10^{9} \mathrm{~s}^{-1}$ is observed. This process is followed by charge separation to yield the oxidized pyrene and reduced perylene chromophores. ${ }^{64}$ The situation is somewhat similar in a supramolecular porphyrin assembly (Figure 5b) reported by Kim and co-workers. ${ }^{65}$ It consists of a tetrameric assembly of pyridineappended porphyrin dyads giving a box composed of eight porphyrin chromophores arranged in a perpendicular geometry. Excitation of any one of the chromophores results in energy transfer occurring intramolecularly (through the alkyne bridge) or intermolecularly through axial ligand coordination. The former is about 10 times faster and it could be shown that the dihedral angle 
a

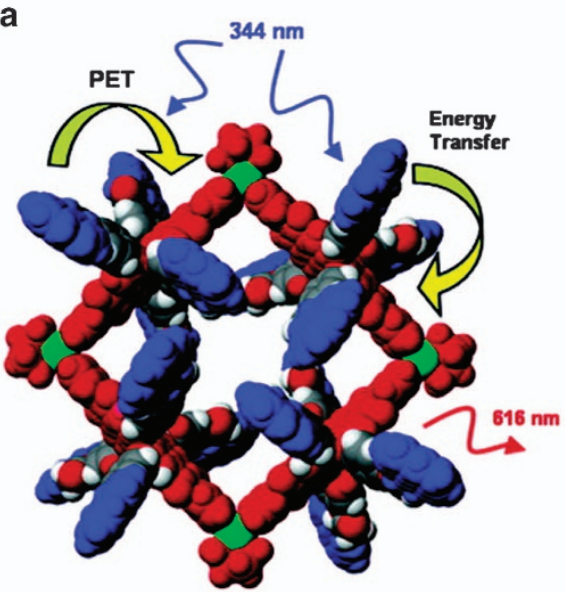

b

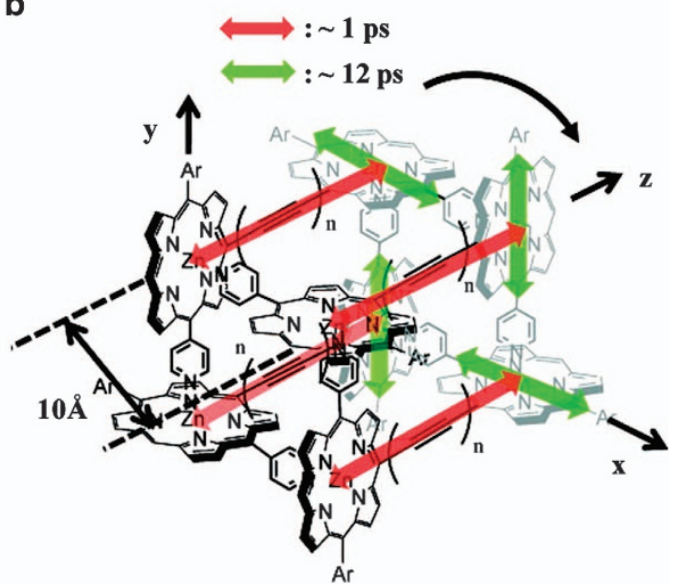

Figure 5 (a) A supramolecular square assembled from four Pt(II) metal centers (green) and four perylene bisimides (red), each of which bears four pyrene units (blue, 16 pyrenes total) shows both photoinduced electron and energy transfer. Reprinted with permission from reference Sautter et al. ${ }^{64}$ Copyright 2005 American Chemical Society. (b) A self-assembled porphyrin cube in which both intramolecular (red arrows) and intermolecular (green arrows) energy transfer is possible. The two differ by ca one order of magnitude in rate. Reprinted with permission from reference Kim et al. ${ }^{65}$ Copyright 2010 American Chemical Society.

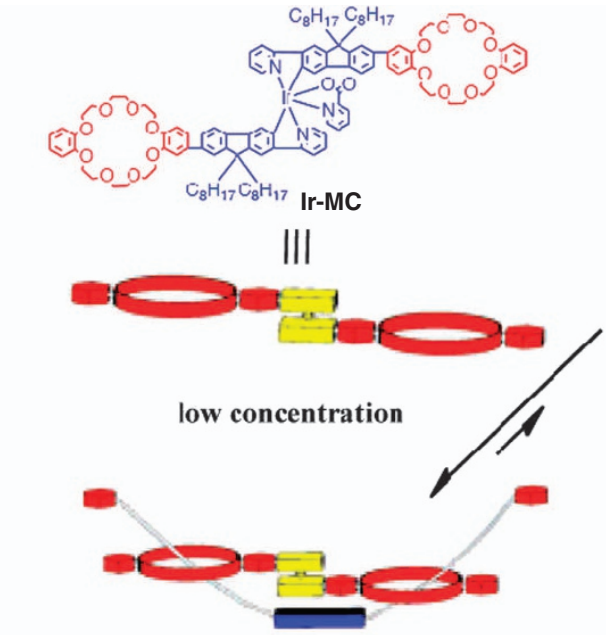

Cyclic species

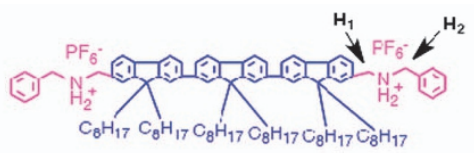

Gt

III

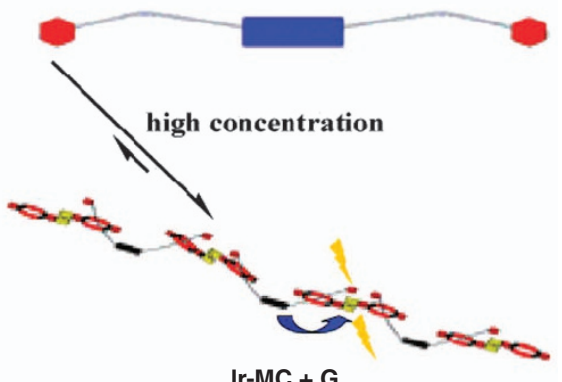

Ir-MC + G

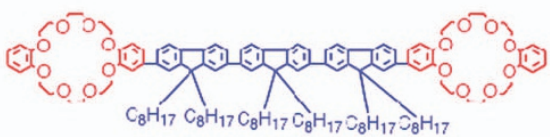

G'
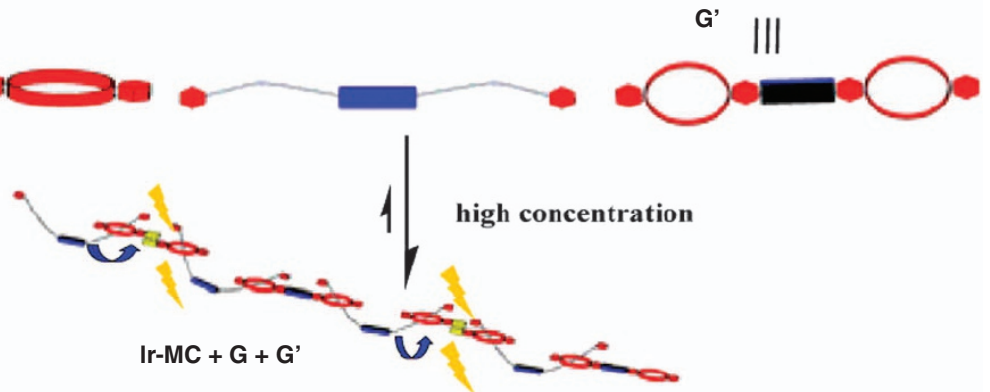

Figure 6 At low concentrations, an iridium complex bearing two crown ether macrocycles (Ir-MC) forms a bis pseudo-rotaxane in the presence of a bis-ammonium guest (G), whereas a supramolecular polymer is obtained at high concentrations. The system displays efficient electroluminescence thanks to intermolecular energy transfer and can be modified with a third component $\left(\mathrm{G}^{\prime}\right.$ ) possessing similar or different electronic properties. Adapted with permission from reference Liang et al. ${ }^{72}$ Copyright 2013 American Chemical Society. 
between the porphyrin units is a decisive factor in determining the excitation energy migration.

Energy transfer in metallo-supramolecular assemblies has been widely used in the construction of photoactive assemblies. In particular, it is of interest for the enhanced excitation of lanthanide luminophores whose absorption cross-section is too low for direct excitation. ${ }^{66-70}$ Irridium complexes are widely used as phosphorescent emissive components in organic light-emitting diode devices, and an elegant approach toward blending them in a solution processable supramolecular polymer was reported by Huang and co-workers. ${ }^{71-73}$ Their approach relies on the reversible formation of pseudo-rotaxanes that are obtained from a bis-macrocyclic iridium complex (Figure 6) and a bis-ammonium terfluorenyl emissive guest (G). At low concentrations, a discreet $1: 1$ assembly is obtained, whereas a supramolecular polymer is formed at high concentrations. The possibility of adding other components $\left(G^{\prime}\right)$ is a particularly appealing advantage of these materials, which afford organic light-emitting diode devices displaying high efficiencies.

Ruthenium polypyridine complexes are another class of metallosupramolecular assembles that have been the focus of considerable attention as they combine the luminescent properties of rutheniu$\mathrm{m}$ (II) centers and good kinetic and thermodynamic stability. ${ }^{74,75}$ Pioneering work on rigid bridges ${ }^{76}$ and dendrimers ${ }^{77}$ rely on an energy gradient to move excitation across the bridge or from the periphery to the core of the dendrimer and are relevant to artificial light-harvesting assemblies. In specific cases, the energy level of an organic excited state is near to that of an inorganic excited state, and equilibration between the two is observed. This is a form of energy transfer that has a result similar to that of delayed luminescence if the two excited states possess widely different decay rates. ${ }^{78}$ By adjusting the energy level of the organic chromophore, it is possible to tailor the system for various metal centers, including more abundant ones that otherwise possess short-lived excited states, such as copper (I) ${ }^{79}$

\section{CONCLUSION AND PERSPECTIVES}

Energy transfer is a fundamental process that is exceedingly well suited to supramolecular architectures, both in view of harnessing the organization they induce and in view of probing it using well-known rate-distance relationships. Controlling the directionality of multistep energy transfer processes within a single architecture remains a challenge. Recent work by Puntoriero et al. ${ }^{80,81}$ in BODIPY assemblies provides an elegant example of ultrafast vectorial energy transfer that occurs on the picosecond time scale. Polysaccharides ${ }^{82}$ and porous silicate frameworks ${ }^{83,84}$ may yet prove to be abundant sources of multivalent scaffolds, which bind chromophores for the construction of light-harvesting architectures. In the assembly of molecular nanowires containing both donor and acceptor fragments, Zhang et al. ${ }^{85}$ showed directionality in the electron transport process, opening the way toward self-assembled molecular rectifiers. Another potential route to well-defined architectures grafted onto solid substrates has been developed by the Matile group, and consists of the surface-initiated supramolecular polymerization process. ${ }^{86-89}$ It has been successfully applied to obtaining redox gradients, and energy transfer across such structures comprising multiple chromophores is expected to be particularly efficient.

\section{ACKNOWLEDGEMENTS}

This work was supported by the National Science Council (Taiwan) (NSC-992923-M-002-002-MY3) and the French ANR (ANR-13-IS07-0001) and LabEx AMADEus (ANR-10-LABX-0042-AMADEUS through grant ANR-10-IDEX0003-02).
1 Burattini, S., Colquhoun, H. M., Greenland, B. W. \& Hayes, W. A novel self-healing supramolecular polymer system. Faraday Discuss. 143, 251-264 (2009).

2 Montarnal, D., Tournilhac, F., Hidalgo, M., Couturier, J. L. \& Leibler, L. Versatile onepot synthesis of supramolecular plastics and self-healing rubbers. J. Am. Chem. Soc. 131, 7966-7967 (2009).

3 Reutenauer, P., Buhler, E., Boul, P. J., Candau, S. J. \& Lehn, J. M. Room temperature dynamic polymers based on Diels-Alder chemistry. Chem. Eur. J. 15, 1893-1900 (2009)

4 Willner, I., Shlyahovsky, B., Zayats, M. \& Willner, B. DNAzymes for sensing, nanobiotechnology and logic gate applications. Chem. Soc. Rev. 37, 1153-1165 (2008)

5 Fukino, T., Joo, H., Hisada, Y., Obana, M., Yamagishi, H., Hikima, T., Takata, M. Fujita, N. \& Aida, T. Manipulation of discrete nanostructures by selective modulation of noncovalent forces. Science 344, 499-504 (2014).

6 Rybtchinski, B. Adaptive supramolecular nanomaterials based on strong noncovalent interactions. ACS Nano 5, 6791-6818 (2011).

7 De Luca, G., Pisula, W., Credgington, D., Treossi, E., Fenwick, O., Lazzerini, G. M. Dabirian, R., Orgiu, E., Liscio, A., Palermo, V., Müllen, K., Cacialli, F. \& Samori, P. Non-conventional processing and post-processing methods for the nanostructuring of conjugated materials for organic electronics. Adv. Funct. Mater. 21, 1279-1295 (2011)

8 Grozema, F. C. \& Siebbeles, L. D. A. Mechanism of charge transport in self-organizing organic materials. Int. Rev. Phys. Chem. 27, 87-138 (2008).

9 Chen, Z. J., Lohr, A., Saha-Moller, C. R. \& Würthner, F. Self-assembled $\pi$-stacks of functional dyes in solution: structural and thermodynamic features. Chem. Soc. Rev. 38, 564-584 (2009).

10 González-Rodríguez, D. \& Schenning, A. P. H. J. Hydrogen-bonded supramolecular $\pi$-functional materials. Chem. Mater. 23, 310-325 (2010).

11 Saha, M. L., De, S., Pramanik, S. \& Schmittel, M. Orthogonality in discrete self-assembly - survey of current concepts. Chem. Soc. Rev. 42, 6860-6909 (2013).

12 Ghosh, S., Li, X. Q., Stepanenko, V. \& Würthner, F. Control of H- and J-type $\pi$ stacking by peripheral alkyl chains and self-sorting phenomena in perylene bisimide homo- and heteroaggregates. Chem. Eur. J. 14, 11343-11357 (2008).

13 Dubacheva, G. V., Liang, C.-K. \& Bassani, D. M. Functional monolayers from carbon nanostructures - fullerenes, carbon nanotubes, and graphene - as novel materials for solar energy conversion. Coord. Chem. Rev. 256, 2628-2639 (2012).

14 Ray, D., Liang, C.-K., McClenaghan, N. D. \& Bassani, D. M. Organic and supramolecular materials for LED and photovoltaic applications. Curr. Phys. Chem. 1, 169-180 (2011).

15 Bassani, D. M., Jonusauskaite, L., Lavie-Cambot, A., McClenaghan, N. D., Pozzo, J.-L., Ray, D. \& Vives, G. Harnessing supramolecular interactions in organic solid-state devices: current status and future potential. Coord. Chem. Rev. 254, 2429-2445 (2010)

16 Babu, S. S., Prasanthkumar, S. \& Ajayaghosh, A. Self-assembled gelators for organic electronics. Angew. Chem. Int. Ed. 51, 1766-1776 (2012).

17 Halls, J. J. M., Pichler, K., Friend, R. H., Moratti, S. C. \& Holmes, A. B. Exciton diffusion and dissociation in a poly(p-phenylenevinylene)/C-60 heterojunction photovoltaic cell. Appl. Phys. Lett. 68, 3120-3122 (1996).

18 Lim, J. M., Kim, P., Yoon, M.-C., Sung, J., Dehm, V., Chen, Z., Würthner, F. \& Kim, D. Exciton delocalization and dynamics in helical $\pi$-stacks of self-assembled perylene bisimides. Chem. Sci. 4, 388-397 (2013).

19 Yagai, S., Usui, M., Seki, T., Murayama, H., Kikkawa, Y., Uemura, S., Karatsu, T., Kitamura, A., Asano, A. \& Seki, S. Supramolecularly engineered perylene bisimide assemblies exhibiting thermal transition from columnar to multilamellar structures. J. Am. Chem. Soc. 134, 7983-7994 (2012).

20 Rao, K. V., Jain, A. \& George, S. J. Organic-inorganic light-harvesting scaffolds for luminescent hybrids. J. Mater. Chem. C 2, 3055-3064 (2014).

21 Ajayaghosh, A., Praveen, V. K. \& Vijayakumar, C. Organogels as scaffolds for excitation energy transfer and light harvesting. Chem. Soc. Rev. 37, 109-122 (2008).

22 Praveen, V. K., Ranjith, C., Bandini, E., Ajayaghosh, A. \& Armaroli, N. Oligo(phenylenevinylene) hybrids and self-assemblies: versatile materials for excitation energy transfer. Chem. Soc. Rev. 43, 4222-4242 (2014).

23 Babu, S. S., Praveen, V. K. \& Ajayaghosh, A. Functional $\pi$-gelators and their applications. Chem. Rev. 114, 1973-2129 (2014).

24 Giansante, C., Raffy, G., Schäfer, C., Rahma, H., Kao, M.-T., Olive, A. G. L. \& Del Guerzo, A. White-light-emitting self-assembled nanofibers and their evidence by microspectroscopy of individual objects. J. Am. Chem. Soc. 133, 316-325 (2011)

25 Giansante, C., Schäfer, C., Raffy, G. \& Del Guerzo, A. Exploiting direct and cascade energy transfer for color-tunable and white-light emission in three-component selfassembled nanofibers. J. Phys. Chem. C 116, 21706-21716 (2012).

26 Del Guerzo, A., Olive, A. G. L., Reichwagen, J., Hopf, H. \& Desvergne, J.-P. Energy transfer in self-assembled [n]-acene fibers involving $\geq 100$ donors per acceptor. J. Am. Chem. Soc. 127, 17984-17985 (2005).

27 Lei, Y.-Y., Jin, Y., Zhou, D.-Y., Gu, W., Shi, X.-B., Liao, L.-S. \& Lee, S.-T. White-light emitting microtubes of mixed organic charge-transfer complexes. Adv. Mater. 24 5345-5351 (2012)

28 Praveen, V. K., Ranjith, C. \& Armaroli, N. White-light-emitting supramolecular gels. Angew. Chem. Int. Ed. 53, 365-368 (2014)

29 Olive, A. G. L., Del Guerzo, A., Schäfer, C., Raffy, G. \& Giansante, C. Fluorescence amplification in self-assembled organic nanoparticles by excitation energy migration and transfer. J. Phys. Chem. C 114, 10410-10416 (2010). 
30 Zhou, Q. \& Swager, T. M. Method for enhancing the sensitivity of fluorescent chemosensors: energy migration in conjugated polymers. J. Am. Chem. Soc. 117, 7017-7018 (1995)

31 Kartha, K. K., Babu, S. S., Srinivasan, S. \& Ajayaghosh, A. Attogram sensing of trinitrotoluene with a self-assembled molecular gelator. J. Am. Chem. Soc. 134, 4834-4841 (2012).

32 Zhang, X., Rehm, S., Safont-Sempere, M. M. \& Würthner, F. Vesicular perylene dye nanocapsules as supramolecular fluorescent $\mathrm{pH}$ sensor systems. Nat. Chem. 1, 623-629 (2009).

33 Zhang, X., Goerl, D. \& Würthner, F. White-light emitting dye micelles in aqueous solution. Chem. Commun. 49, 8178-8180 (2013).

34 Goerl, D., Zhang, X. \& Würthner, F. Molecular aggregates of perylene bisimide dyes in water. Angew. Chem. Int. Ed. 51, 6328-6348 (2012).

35 Yagai, S., Aonuma, H., Kikkawa, Y., Kubota, S., Karatsu, T., Kitamura, A., Mahesh, S. \& Ajayaghosh, A. Rational design of nanofibers and nanorings through complementary hydrogen-bonding interactions of functional $\pi$ systems. Chem. Eur. J. 16, 8652-8661 (2010).

36 Kuo, M.-C., Chen, H.-F., Shyue, J.-J., Bassani, D. M. \& Wong, K.-T. In situ reversible conversion of porphyrin aggregate morphology. Chem. Commun. 48, 8051-8053 (2012).

37 Tseng, K.-P., Tsai, Y.-T., Wu, C.-C., Shyue, J.-J., Bassani, D. M. \& Wong, K.-T. Lightand solvent-driven morphological transformations of self-assembled hydrogen-bonded nanostructures. Chem. Commun. 49, 11536-11538 (2013).

38 Dautel, O. J., Robitzer, M., Flores, J.-C., Tondelier, D., Serein-Spirau, F., Lère-Porte, J.-P., Guérin, D., Lenfant, S., Tillard, M., Vuillaume, D. \& Moreau, J. J. E. Electroactive nanorods and nanorings designed by supramolecular association of $\pi$-conjugated oligomers. Chem. Eur. J 14, 4201-4213 (2008).

39 Tseng, K.-P., Fang, F.-C., Shyue, J.-J., Wong, K.-T., Raffy, G., Del Guerzo, A. \& Bassani, D. M. Spontaneous generation of highly emissive RGB organic nanospheres. Angew. Chem. Int. Ed. 50, 7032-7036 (2011).

40 Velu, S. K. P., Yan, M., Tseng, K.-P., Wong, K.-T., Bassani, D. M. \& Terech, P. Spontaneous formation of artificial vesicles in organic media through hydrogen-bonding interactions. Macromolecules 46, 1591-1598 (2013).

41 Rao, K. V., Datta, K. K. R., Eswaramoorthy, M. \& George, S. J. Highly pure solid-state white-light emission from solution-processable soft-hybrids. Adv. Mater. 25, 1713-1718 (2013).

42 Seki, T., Maruya, Y., Nakayama, K.-i., Karatsu, T., Kitamura, A. \& Yagai, S. Solution processable hydrogen-bonded perylene bisimide assemblies organizing into lamellar architectures. Chem. Commun. 47, 12447-12449 (2011).

43 Stevens, A. L., Kaeser, A., Schenning, A. P. H. J. \& Herz, L. M. Morphology-dependent energy transfer dynamics in fluorene-based amphiphile nanoparticles. ACS Nano 6, 4777-4787 (2012).

44 Ajayaghosh, A., Vijayakumar, C., Praveen, V. K., Babu, S. S. \& Varghese, R. Selflocation of acceptors as "isolated" or "stacked" energy traps in a supramolecular donor self-assembly: a strategy to wavelength tunable FRET emission. J. Am. Chem. Soc. 128, 7174-7175 (2006)

45 Hennig, A., Hatami, S., Spieles, M. \& Resch-Genger, U. Excitation energy migration and trapping on the surface of fluorescent poly(acrylic acid)-grafted polymer particles. Photochem. Photobiol. Sci. 12, 729-737 (2013).

46 Fink, R. F., Seibt, J., Engel, V., Renz, M., Kaupp, M., Lochbrunner, S., Zhao, H. M., Pfister, J., Würthner, F. \& Engels, B. Exciton trapping in $\pi$-conjugated materials: a quantum-chemistry-based protocol applied to perylene bisimide dye aggregates. J. Am Chem. Soc. 130, 12858-11259 (2008).

47 Kwon, J. E., Park, S. \& Park, S. Y. Realizing molecular pixel system for full-color fluorescence reproduction: RGB-emitting molecular mixture free from energy transfer crosstalk. J. Am. Chem. Soc. 135, 11239-11246 (2013).

48 West, B. A., Womick, J. M., McNeil, L. E., Tan, K. J. \& Moran, A. M. Ultrafast dynamics of frenkel excitons in tetracene and rubrene single crystals. J. Phys. Chem. C 114 10580-10591 (2010).

49 Bu, L., Pentzer, E., Bokel, F. A., Emrick, T. \& Hayward, R. C. Growth of polythiophene/ perylene tetracarboxydiimide donor/acceptor shish-kebab nanostructures by coupled crystal modification. ACS Nano 6, 10924-10929 (2012).

50 Sukhanova, A., Baranov, A. V., Perova, T. S., Cohen, J. H. M. \& Nabiev, I. Controlled self-assembly of nanocrystals into polycrystalline fluorescent dendrites with energy-transfer properties. Angew. Chem. Int. Ed. 45, 2048-2052 (2006).

51 Li, H., Babu, S. S., Turner, S. T., Neher, D., Hollamby, M. J., Seki, T., Yagai, S., Deguchi, Y., Möhwald, H. \& Nakanishi, T. Alkylated-C60 based soft materials: regulation of self-assembly and optoelectronic properties by chain branching. J. Mater. Chem. C 1, 1943-1951 (2013).

52 Chu, C.-C., Raffy, G., Ray, D., Del Guerzo, A., Kauffmann, B., Wantz, G., Hirsch, L. \& Bassani, D. M. Self-assembly of supramolecular fullerene ribbons via hydrogenbonding interactions and their impact on fullerene electronic interactions and charge carrier mobility. J. Am. Chem. Soc. 132, 12717-12723 (2010).

53 Raffy, G., Ray, D., Chu, C.-C., Del Guerzo, A. \& Bassani, D. M. Controlling the emission polarization from single crystals using light: towards photopolic materials. Angew. Chem. Int. Ed. 50, 9584-9588 (2011).

54 Darcos, V., Griffith, K., Sallenave, X., Desvergne, J.-P., Guyard-Duhayon, C., Hasenknopf, B. \& Bassani, D. M. Supramolecular control of [2+2] photodimerization via hydrogen bonding. Photochem. Photobiol. Sci. 2, 1152-1161 (2003).

55 Raffy, G., Ray, D., Chu, C.-C., Del Guerzo, A. \& Bassani, D. M. Effect of hydrogenbonding on the excited-state reactivity of fullerene derivatives and its impact on the control of the emission polarisation from photopolic single crystals. Phys. Chem. Chem. Phys. 14, 8859-8865 (2012).

56 Guldi, D. M. Fullerene-porphyrin architectures; photosynthetic antenna and reaction center models. Chem. Soc. Rev. 31, 22-36 (2002).

57 Babu, S. S., Möhwald, H. \& Nakanishi, T. Recent progress in morphology control of supramolecular fullerene assemblies and its applications. Chem. Soc. Rev. 39, 4021-4035 (2010).

58 D'Souza, F. \& Ito, O. Photoinduced electron transfer in supramolecular systems of fullerenes functionalized with ligands capable of binding to zinc porphyrins and zinc phthalocyanines. Coord. Chem. Rev. 249, 1410-1422 (2005).

59 Chandra, B., Stranius, K., D'Souza, P., Subbaiyan, N. K., Lemmetyinen, H., Tkachenko, N. V. \& D'Souza, F. Sequential photoinduced energy and electron transfer directed improved performance of the supramolecular solar cell of a zinc porphyrin-zinc phthalocyanine conjugate modified $\mathrm{TiO}_{2}$ surface. J. Phys. Chem. C 117, 763-773 (2013).

60 Kira, A., Tanaka, M., Umeyama, T., Matano, Y., Yoshimoto, N., Zhang, Y., Ye, S., Lehtivuori, H., Tkachenko, N. V., Lemmetyinen, H. \& Imahori, H. Hydrogen-bonding effects on film structure and photoelectrochemical properties of porphyrin and fullerene composites on nanostructured $\mathrm{TiO}_{2}$ electrodes. J. Phys. Chem. C 111, 13618-13626 (2007)

61 KC, C. B., Ohkubo, K., Karr, P. A., Fukuzumi, S. \& D'Souza, F. Two-point bound zinc porphyrin-zinc phthalocyanine-fullerene supramolecular triad for sequential energy and electron transfer. Chem. Commun. 49, 7614-7616 (2013).

62 D'Souza, F., Amin, A. N., El-Khouly, M. E., Subbaiyan, N. K., Zandler, M. E. \& Fukuzumi, S. Control over photoinduced energy and electron transfer in supramolecular polyads of covalently linked azabodipy-bisporphyrin 'molecular clip' hosting fullerene. J. Am. Chem. Soc. 134, 654-664 (2012).

63 Nandwana, V., Serrano, L. A., Solntsev, K. M., Ebenhoch, B., Liu, Q., Tonga, G. Y., Samuel, I. D. W., Cooke, G. \& Rotello, V. M. Engineering the nanoscale morphology of a quantum dot-fullerene assembly via complementary hydrogen bonding interactions. Langmuir. 29, 7534-7537 (2013).

64 Sautter, A., Kaletas, B. K., Schmid, D. G., Dobrawa, R., Zimine, M., Jung, G., Van Stokkum, I. H. M., De Cola, L., Williams, R. M. \& Würthner, F. Ultrafast energy-electron transfer cascade in a multichromophoric light-harvesting molecular square. J. Am. Chem. Soc. 127, 6719-6729 (2005).

65 Kim, P., Lim, J. M., Yoon, M.-C., Aimi, J., Aida, T., Tsuda, A. \& Kim, D. Excitation energy migration processes in self-assembled porphyrin boxes constructed by conjugated porphyrin dimers. J. Phys. Chem. B 114, 9157-9164 (2010).

66 Kawa, M. \& Frechet, J. M. J. Self-assembled lanthanide-cored dendrimer complexes: enhancement of the luminescence properties of lanthanide ions through site-isolation and antenna effects. Chem. Mater. 10, 286-296 (1998).

67 Moore, E. G., Samuel, A. P. S. \& Raymond, K. N. From antenna to assay: lessons learned in lanthanide luminescence. Acc. Chem. Res. 42, 542-552 (2009).

68 Armelao, L., Quici, S., Barigelletti, F., Accorsi, G., Bottaro, G., Cavazzini, M. \& Tondello, E. Design of luminescent lanthanide complexes: from molecules to highly efficient photoemitting materials. Coord. Chem. Rev. 254, 487-505 (2010).

69 Alpha, B., Ballardini, R., Balzani, V., Lehn, J. M., Perathoner, S. \& Sabbatini, N. Antenna effect in luminescent lanthanide cryptates: a photophysical study. Photochem. Photobiol. 52, 299-306 (1990).

70 Balzani, V., Bergamini, G., Campagna, S. \& Puntoriero, F. Photochemistry and photophysics of coordination compounds: overview and general concepts. Top. Curr. Chem. 280, 1-36 (2007)

71 Zhang, J., Zhang, K., Liu, S., Liang, A., Huang, X., Huang, F., Peng, J. \& Cao, Y. A supramolecular large band gap host for phosphorescent organic light-emitting diodes. RSC Adv. 3, 3829-3835 (2013).

72 Liang, A.-H., Zhang, K., Zhang, J., Huang, F., Zhu, X.-H. \& Cao, Y. Supramolecular phosphorescent polymer iridium complexes for high-efficiency organic light-emitting diodes. Chem. Mater. 25, 1013-1019 (2013).

73 Liang, A.-h., Dong, S., Zhang, K., Xiao, X., Huang, F., Zhu, X.-H. \& Cao, Y. Supramolecular sky-blue phosphorescent polymer iridium complexes for single-emissive-layer organic light-emitting diodes. Macromol. Rapid Commun. 34, 1301-1305 (2013).

74 Juris, A., Balzani, V., Barigelletti, F., Campagna, S., Belser, P. \& Von Zelewsky, A. Ruthenium(II) polypyridine complexes: photophysics, photochemistry, electrochemistry, and chemiluminescence. Coord. Chem. Rev. 84, 85-277 (1988).

75 Balzani, V., Juris, A., Venturi, M., Campagna, S. \& Serroni, S. Luminescent and redoxactive polynuclear transition metal complexes. Chem. Rev. 96, 759-833 (1996).

76 De Cola, L. \& Belser, P. Photoinduced energy- and electron transfer processes in rigidly bridged dinuclear Ru/Os complexes. Coord. Chem. Rev. 177, 301-346 (1998)

77 Balzani, V., Campagna, S., Denti, G., Juris, A., Serroni, S. \& Venturi, M. Designing dendrimers based on transition-metal complexes. Light-harvesting properties and predetermined redox patterns. Acc. Chem. Res. 31, 26-34 (1998).

78 Passalacqua, R., Loiseau, F., Campagna, S., Fang, Y.-Q. \& Hanan, G. S. In search of ruthenium(II) complexes based on tridentate polypyridine ligands that feature long-lived room-temperature luminescence: the multichromophore approach. Angew. Chem. Int. Ed. 42, 1608-1611 (2003).

79 Leydet, Y., Bassani, D. M., Jonusauskas, G. \& McClenaghan, N. D. Equilibration between three different excited states in a bichromophoric copper(I) polypyridine complex. J. Am. Chem. Soc. 129, 8688-8689 (2007).

80 Bura, T., Nastasi, F., Puntoriero, F., Campagna, S. \& Ziessel, R. Ultrafast energy transfer in triptycene-grafted bodipy scaffoldings. Chem. - Eur. J. 19, 8900-8912 (2013). 
81 Puntoriero, F., Nastasi, F., Campagna, S., Bura, T. \& Ziessel, R. Vectorial photoinduced energy transfer between boron-dipyrromethene (Bodipy) chromophores across a fluorene bridge. Chem. - Eur. J. 16, 8832-8845 (2010).

82 Bonaccorsi, P., Aversa, M. C., Barattucci, A., Papalia, T., Puntoriero, F. \& Campagna, S. Artificial light-harvesting antenna systems grafted on a carbohydrate platform. Chem. Commun. 48, 10550-10552 (2012).

83 Cucinotta, F., Carniato, F., Devaux, A., De Cola, L. \& Marchese, L. Efficient photoinduced energy transfer in a newly developed hybrid SBA-15 photonic antenna. Chem. Eur. J. 18, 15310-15315 (2012).

84 Ruiz, A. Z., Li, H. \& Calzaferri, G. Organizing supramolecular functional dye-zeolite crystals. Angew. Chem. Int. Ed. 45, 5282-5287 (2006).

85 Zhang, W., Jin, W., Fukushima, T., Saeki, A., Seki, S. \& Aida, T. Supramolecular linear heterojunction composed of graphite-like semiconducting nanotubular segments. Science 334, 340-343 (2011).

86 Sforazzini, G., Orentas, E., Bolag, A., Sakai, N. \& Matile, S. Toward oriented surface architectures with three coaxial charge-transporting pathways. J. Am. Chem. Soc. 135, 12082-12090 (2013).

87 Sakurai, S.-i., Areephong, J., Bertone, L., Lin, N.-T., Sakai, N. \& Matile, S. Toward polymerized artificial photosystems with supramolecular $\mathrm{n} / \mathrm{p}$-heterojunctions and antiparallel redox gradients. Energy Environ. Sci. 4, 2409-2416 (2011).
88 Bhosale, R., Misek, J., Sakai, N. \& Matile, S. Supramolecular n/p-heterojunction photosystems with oriented multicolored antiparallel redox gradients (OMARG-SHJs). Chem. Soc. Rev. 39, 138-149 (2010).

89 Bhosale, R., Perez-Velasco, A., Ravikumar, V., Kishore, R. S. K., Kel, O., Gomez Casado, A., Jonkheijm, P., Huskens, J., Maroni, P., Borkovec, M., Sawada, T., Vauthey, E., Sakai, N. \& Matile, S. Topologically matching supramolecular n/p-heterojunction architectures. Angew. Chem. Int. Ed. 48, 6461-6464 (2009).

(c) (1) () $\odot$ This work is licensed under a Creative Commons Attribution-NonCommercial-NoDerivs 3.0 Unported License. The images or other third party material in this article are included in the article's Creative Commons license, unless indicated otherwise in the credit line; if the material is not included under the Creative Commons license, users will need to obtain permission from the license holder to reproduce the material. To view a copy of this license, visit http://creativecommons.org/licenses/by-nc-nd/3.0/

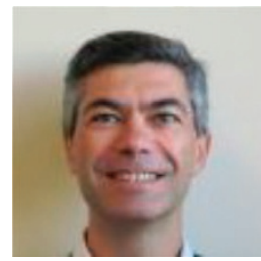

Dario Bassani obtained his PhD from Northwestern University under the guidance of FD Lewis and was postdoctoral fellow with J Wirz (University of Basle) and J-M Lehn (University of Strasbourg) before joining the Centre National de la Recherche Scientifique at the University of Bordeaux. His research interests include supramolecular photochemistry and photoactive materials for optoelectronic applications.
Dr Ken-Tsung Wong obtained his PhD degree from National Taiwan University in 1993. He conducted his postdoctoral researches at University of Illinois at Urbana-Champaign in 1995-1996 and Université Louis Pasteur, Strasbourg in 1996-1998 before he joined the Department of Chemistry at National Taiwan University as an assistant professor. He was promoted to an associate professor 4 years later and professor in 2006. He established a strong interdisciplinary research program engaging domestic and international collaborations. His research mainly focuses on the molecular design and organic synthesis of novel $\pi$-conjugated and supramolecular materials for optoelectronic applications such as organic light-emitting diode (OLED), electrogenerated chemiluminescence (ECL), solid-state light-emitting electrochemical cells (LEC) and photovoltaic devices. 